\title{
TRANSLOCACIÓN ROBERTSONIANA ENTRE LOS CROMOSOMAS (No13/15): A PROPÓSITO DE UN CASO,HOSPITAL LUIS G DÁVILA DE TULCÁN, AÑO 2016
}

ROBERTSONIAN TRANSLOCATION BETWEEN CHROMOSOMES (No13 / 15): A CASE REPORT IN THE LUIS G DÁVILA HOSPITAL IN TULCÁN, IN THE YEAR 2016

\author{
TRANSLOCAÇÃO ROBERTSONIANA ENTRE CROMOSSOMOS (N013/ 15): \\ SOBRE UM CASO DO HOSPITAL LUIS G DÁVILA EM TULCÁN, ANO 2016
}

\author{
VICTOR GUAPI NAUÑAY', GRISELDA DE LA CRUZ JIMÉNEZ', SANDRA MERA BASTIDAS' \\ 1 Hospital Luis Gabriel Dávila, Tulcán, Ecuador \\ 2 Universidad Católica de Santiago de Guayaquil; Guayaquil, Ecuador
}

RESUMEN

Introducción: la translocación robertsoniana, se define como la fusión de dos cromosomas acrocéntricos no homólogos, con una frecuencia de 1 caso por cada 1000 recien nacidos. Objetivo: establecer el efecto clinico de la translocación Robertsoniana y describir el complemento cromosómico del gameto de la portadora. Materiales: estudio observacional descriptivo de tipo reporte de caso. Como criterios de inclusión se consideró el antecedente de abortos recurrentes con fenotipo normal y estudio citogenético con translocación. Los hallazgos fueron redactados usando el software Microff Office Professional Plus 2013. Resultados: paciente de sexo femenino de 31 años de edad, con los siguientes antecedentes gineco-obstètricos: gestas: 7, abortos 6, partos 0 , cesáreas 1 , hijos vivos 1 , hijos muertos 0 . Gesta 1: hija de 12 años de edad, con ausencia de dismorfias, desde la segunda gesta hace 11 años hasta la gesta siete ocurrida en el presente año, han terminado en abortos espontáneos antes de las 12 primeras semanas de gestación. Con estudio citogenético que reporta translocación Robetsoniana, 45, XX, t(13/15). Discusión: la translocación robetsoniana ocurre por la rotura de dos cromosomas acrocéntricos, dando como resultado un cariotipo equilibrado con 45 cromosomas, incluido el cromosoma translocado, formado por el brazo largo de los cromosomas 13 y 15. Entre los gametos que la heterocigota portadora puede producir, estan los desequilibrados, quienes generan descendencia no viable, acontecimiento que explica en la probando las pérdidas precoses de los embarazos. Conclusión: el portador de una translocación Robertsoniana entre los cromosomas 13/15 tiene solo 45 cromosomas, acontecimiento que conduce a la pérdica precoz del embarazo ó al nacimiento de un neonato con múltiples defectos. PALABRAS CLAVE: aborto habitual, translocación genética, cariotipo.

ABSTRACT

Introduction: the Robertsonian translocation is defined as the fusion of two non-homologous acrocentric chromosomes, with a frequency of 1 case per 1,000 newborns. Objective: establish the clinical effect of Robertsonian translocation and describe the chromosomal complement of the carrier's gamete. Materials: a descriptive observational study of a case report type. The inclusion criteria were a history of recurrent abortions with a normal phenotype and a cytogenetic study with translocation. The findings were written using Microsoft Office Professional Plus 2013 software. Results: 31-year-0ld female patient, with the following gynecological and obstetric antecedents: gestations: 7, abortions 6, natural births 0 , cesarean sections 1 , live children 1, dead children 0 . Gestation 1: 12-year-old daughter, with the absence of dysmorphia, from the second gestation 11 years ago to the seventh gestation that occurred this year, have ended in spontaneous abortions before the first 12 weeks of gestation. With a cytogenetic study that reports Robertsonian translocation, 45, XX, t (13/15). Discussion: Robertsonian translocation occurs due to the breakage of two acrocentric chromosomes, resulting in a balanced karyotype with 45 chromosomes, including the translocated chromosome, formed by the long arm of chromosomes 13 and 15 . Among the gametes that the heterozygous carrier can produce are the unbalanced ones, that generate non-viable offspring, an event that explains the early pregnancy loss in the trial. Conclusion: the Robertsonian translocation carrier between chromosomes $13 / 15$ has only 45 chromosomes, an event that leads to early loss of a pregnancy or the birth of a newborn with multiple defects.

KEYWORDS: abortion habitual, translocation genetic, caryotype.

RESUMO

Introdução: a translocação robertsoniana é definida como a fusão de dois cromossomos acrocêntricos não homólogos, com frequência de 1 caso por 1.000 recém-nascidos. Objetivo: estabelecer 0 efeito clínico da translocação robertsoniana e descrever o complemento cromossômico do gameta do portador. Materiais: estudo observacional descritivo do tipo relato de caso. Os critérios de inclusão foram história de abortos recorrentes com fenótipo normal e estudo citogenético com translocação. Os achados foram escritos usando o software Microff Office Professional Plus 2013. Resultados: paciente do sexo feminino, 31 anos, com a seguinte história ginecológica-obstétrica: gestações: 7, abortos 6, partos 0, cesarianas 1, filhos vivos 1, 0 filhos mortos Gesta 1: filha de 12 anos, com ausência de dismorfia, desde a segunda gestação há 11 anos até a 7 gestação ocorrida neste ano, terminaram em aborto espontâneo antes das primeiras 12 semanas de gestação. Com estudo citogenético que relata translocação robetsoniana, 45, XX, t (13/15). Discussão: a translocação robetsoniana ocorre devido à quebra de dois cromossomos acrocêntricos, resultando em um cariótipo balanceado com 45 cromossomos, incluindo 0 cromossomo translocado, formado pelo braço longo dos cromossomos 13 e 15 . Dentre os gametas que o portador heterozigoto pode produzir existem os desequilibrados, que geram descendentes inviáveis, evento que explica a perda precoce de gestações no ensaio. Conclusão: 0 portador de uma translocação robertsoniana entre os cromossomos $13 / 15$ possui apenas 45 cromossomos, evento que leva à perda precoce da gravidez ou ao nascimento de um recém-nascido com múltiplos defeitos.

PALAVRAS-CHAVE: aborto habitual, translocação genética. 


\section{INTRODUCCIÓN}

Entre las cromosomopatías estructurales esta la translocación Robertsoniana, definida como la fusión de dos cromosomas acrocentricos no homologos..$^{1-3}$ La frecuencia es 1 caso por cada 1 000 recien nacidos. ${ }^{4,5}$ Ocurre con los cinco cromosomas acrocentricos 13, 14, 15, 21 y 22 que tienen un brazo corto. ${ }^{6}$

La translocación es observada en cariotipo convencional y puede reducirse el numero de cromosomas si el brazo corto del cromosoma producto de la translocación se pierde en la división celular. ${ }^{7,8}$ Las personas portadoras de una translocación Robertsoniana son fenotipicamente normales, ${ }^{9,10}$ El instante en que desean concebir pueden encontrar dificultad puesto que presentan historia de infertilidad, abortos espontaneos, muertes fetales e hijos con aberraciones cromosómicas no balanceadas. ${ }^{11,12}$

METODOLOGÍA

Estudio observacional descriptivo de tipo reporte de caso. Como criterios de inclusión se consideró el antecedente de abortos recurrentes con fenotipo normal y estudio citogenético con translocación; cumpliendo con los criterios un caso de la consulta externa de Asesoramiento Genético del hospital provincial Luis G Dávila, en el año 2016.

Se realizó una exhaustiva anamnesis y examen fisico de la paciente, contrastando con el análisis de los exámenes de gabinete. Los hallazgos fueron redactados usando el software Microff Office Professional Plus 2013, y para el diseño del complemento cromosómico se uso el software Visio 2013. Además se obtuvo el consentimiento informado de la paciente para realizar estudio citogenético.

En la presente investigación médica, se protege la vida, la salud, la dignidad, la integridad, el derecho a la autodeterminación, la intimidad y la confidencialidad de la información personal de la paciente que participa en la investigación. Ademàs se envió el protocolo de investigación al Comité de ética de investigación, para su comentario, consejo y aprobación pertinente.

Se brindo una amplica información a la paciente acerca del objetivo de la presente investigación y después de asegurarnos que comprendio, se pidio por escrito el consentimiento informado de manera voluntaria, incluso se consulto al esposo sobre la participación.

\section{CASO CLÍNICO}

Paciente de sexo femenino de 31 años de edad, es referida del hospital básico de San Gabriel (11/09/2016) por primera vez al servicio de emergencia, àrea de Cinecología en el hospital provincial Luis G Dávila, por aborto imcompleto. Con normas de asepcia y anticepcia, bajo anestesia general se realizo Aspiración Manual Endometrial (AMEU), sin complicación alguna.

Retorna la paciente a la consulta externa de Cinecología (21/09/2016), donde se realizo examenes de autoinmunidad: antifosfolipidos IgM $<10 \mathrm{U} / \mathrm{ml}$, anticoagulante lupico 30 segundos, cardiolipina IgM $<7 \mathrm{U} / \mathrm{ml}$, resultando todos negativos.

La paciente fue remitida a la consulta (23/10/2016) de Asesoramiento Genético por antecedente de seis abortos ocurridos en el primer trimestre de embarazo. En esta consulta se realizó una exhaustiva anamnesis, confección del familiograma y examen fisico de la paciente, con los hallazgos a continuación descritos: talla $1.62 \mathrm{~cm}$, peso 83.3 $\mathrm{kg}$, BMI 32.13, tensión arterial 116/76 mmHg, frecuencia cardiaca 98 latidos por minuto, frecuencia respiratoria 20 por minuto y temperatura 36 , $1{ }^{\circ} \mathrm{C}$. Fenotipo femenino normal, con inteligencia consevada.

Es la primera hija de dos hijas de una pareja sin historia de consanguinidad o endogamia. Con crecimiento adecuado en la niñez e instrucción primaria completa.

Con los siguientes antecedentes gineco-obstétricos: menarquia a los once años, ciclos menstruales regulares, con menstruación por cuatro días. Gestas: 7, abortos 6, partos 0 , cesàreas 1 , hijos vivos 1, hijos muertos 0 .

Gesta 1: hija de 12 años de edad, con ausencia de dismorfias, fue obtenida por cesàrea. Desde la segunda gesta hace 11 años hasta la gesta siete ocurrida en el presente año, han terminado en abortos espontáneos antes de las 12 primeras semanas de gestación.

Los estudios de laboratorio reportan: hemograma y bioquímica normal, glucosa en ayunas: $89 \mathrm{mg} /$ dl, con TSH: 2,1 mU/L y T4L: $1.2 \mathrm{ng} / \mathrm{dL}$. El rastreo ecogradico de la cavidad uterina no evidencia anomalias anatomicas, con anexo derecho e izquierdo normal. 


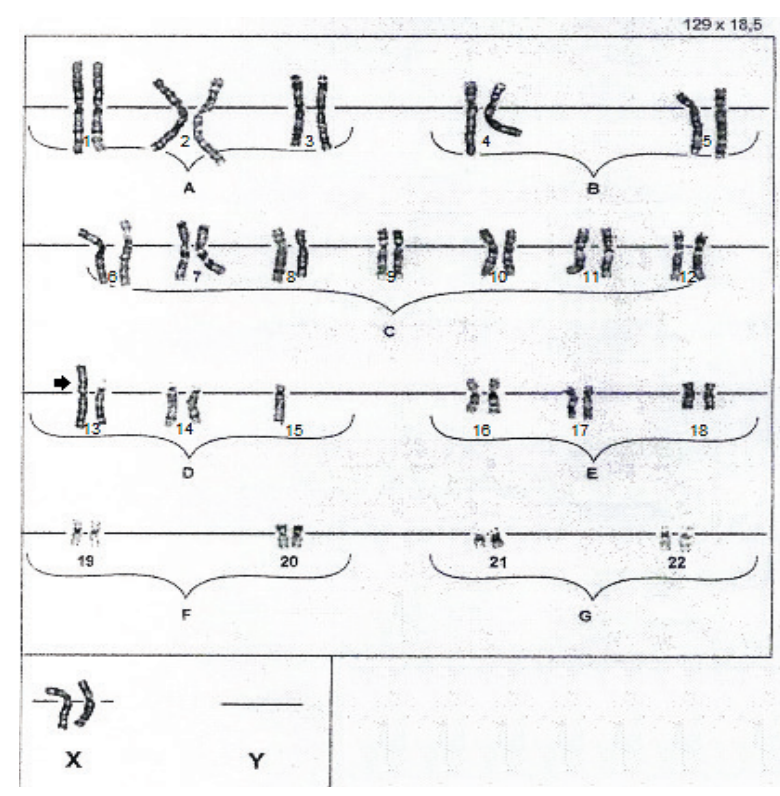

Figura 1. Cariotipo con translocación Robetsoniana, 45, XX, t(13/15)

Después de obtener el concentimiento informado de la probando, se procedió a tomar una muestra de $3 \mathrm{ml}$ de sangre periférica, en un tubo esteril heparinizado de tal manera que se impida la coagulación y puedan mantenerse libres los linfocitos. ${ }^{13} \mathrm{El}$ medio de cultivo fue enriquecido con suplemento exógeno, proporcionando a las células condiciones favorables con nutrientes y factores de crecimiento que permitió iniciar y mantener suficientes ciclos celulares in vitro. ${ }^{11,14}$

Los linfocitos $\mathrm{T}$, fueron estimulados con fibrohemaglutinina (FHA), e incubados a $37^{\circ} \mathrm{C}$ durante 72 horas. El cultivo estuvo tratado con colchicina, que inhibio la formacion del huso cromático e incubamos a $37^{\circ} \mathrm{C}$ por varios minutos. La cosecha de 20 metafases se realizó una vez detenido el cultivo en metafase. Procedimos a realizar una primera centrifugación, posteriormente estraimos el sobrante que es sustituido por igual cantidad de una solución hipotónica y realizamos la segunda centrifugación.

Para la fijación se utilizó ácido acético y metanol, con extensión sobre una làmina portaobjeto, ademàs de la coloración con tripsina-giemsa. En la observación bajo el microscopio óptico, cada cromosoma fue analizado considerando la presencia y la pareja de los cromosomas humanos en orden bien definido. El resultado de nuestra probando de esta familia correspondio a una translocación Robertsoniana entre los cromosomas №¹3/15. (Figura 1)
DISCUSIÓN

Una translocación Robertsoniana ocurre por la rotura de dos cromosomas acrocéntricos (números $13,14,15,21$ y 22) en o cerca de los centrómeros, con la posterior fusión de sus brazos largos. ${ }^{14,15}$ Como no hay pérdida ni ganancia de material genético importante, se considera una reconfiguración funcionalmente equilibrada. ${ }^{15,16}$

El cariotipo equilibrado resultante (II-7) tiene 45 cromosomas con el cromosoma translocado que, de hecho està formado por los brazos de los cromosomas 13 y 15.

Por otro lado, los brazos cortos de los cromosomas involucrados en la fusión a menudo se pierden. Estos brazos al parecer no tienen importancia clínica, ${ }^{(17,18)}$ puesto que solo tienen genes de RNA ribosómico, para el cual existen múltiples copias en los demàs cromosomas acrocéntricos. ${ }^{15}$

Las translocaciones Robertsonianas pueden ser monocéntricas o seudodicéntricas. ${ }^{17}$ La localización del punto de ruptura en el presente caso, fue en el centromero; esta unión se denomina fusión centrica. ${ }^{15}$

Miryounessi $M$, et al ${ }^{5,17,19}$ públicó que entre las translocaciones Robertsonianas màs frecuentes estubieron el 13q14qy 14q21q; mientras que entre el reordenamiento cromosómico menos común ${ }^{20}$ con el $4.12 \%$ se encuentra la $t(13 / 15)$, presente en nuestro caso.

La mayoria de los heterocigotos de translocación Robetsoniana se heredan a partir de un padre ó madre portador, mientras que la minoría son de novo resultado que se ha generado en la meiosis I de la ovogénesis. ${ }^{11,19}$

La presencia de una hija heterocigota portadora (II-7), fenotipicamente normal en la segunda generación del familiograma (Figura 2) identifica al hombre I- 5 o a la mujer I- 6 como portadores; en general, las mujeres portadoras muestran un riesgo incrementado de transmitir la translocación a su descendencia, ${ }^{17}$ situación que se definira con el estudio citogenético.

Los abortos (III-10/III-16) de la pareja II-6 y II-7, crean una insertidumbre a conocer cual de los padres es el portador de la translocación, es asi que el hombre II- 6 , presenta un cariotipo normal, $46, \mathrm{XY}$. 


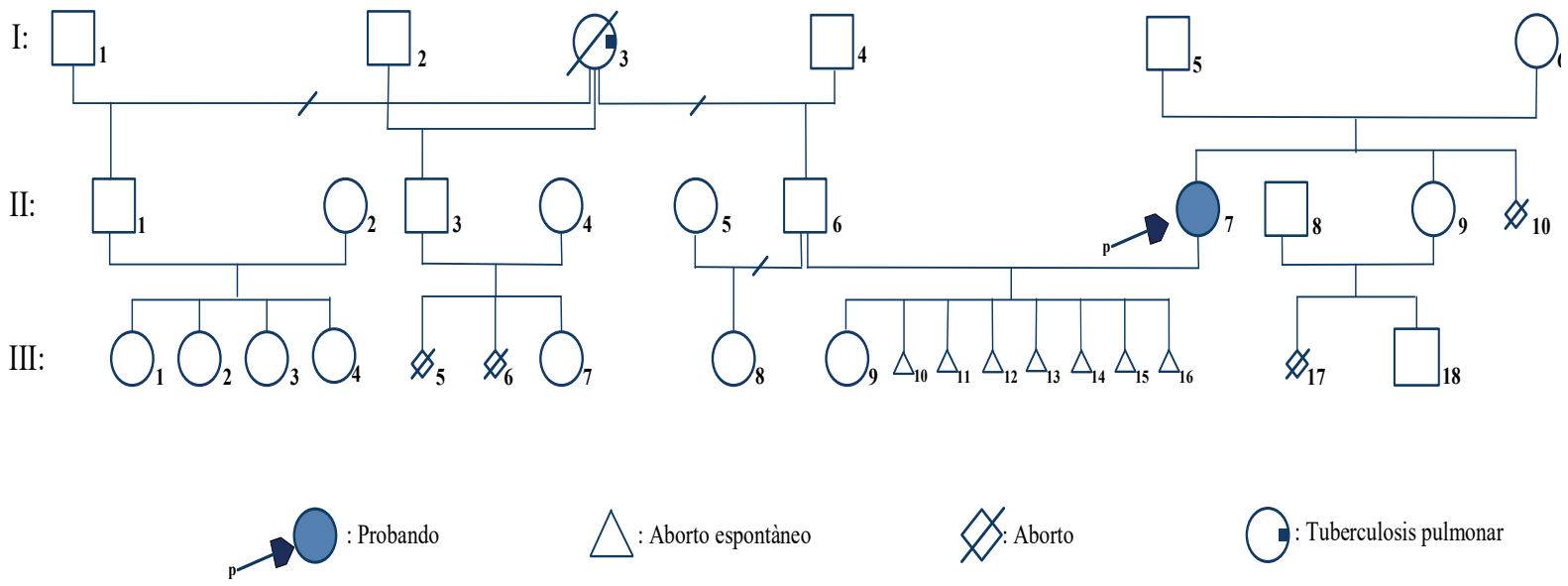

Figura 2. Familiograma con el probando que posee las translocaciòn

Los gametos que la heterocigota portadora puede producir, a continuación son descritos: 1.Un complemento cromosómico normal, es decir un 13 y un 15 normal. 2. Un complemento cromosómico equilibrado, dado por el cromosoma con la translocación 13/15. . $5,17,21$ Gametos que son viable, muestra de ello es la presencia de III-9, en en la tercera generación del familiograma, con la interrogante si es portadora igual que su madre, fenotípicamente normal. 3. Un cromosoma desequilibrado del gameto, que posea el cromosoma con la traslocación 13/15 màs el 13 normal; esto descencadenaria que el embrión fertilizado presente un trisomía 13. 4. El complemento cromosómico desequilibrado con el 13 normal y ausencia del un 15. 5. Un complemento cromosómico desequilibrado con el cromosoma 13/15 de la translocación y un cromosoma 15 normal.
6. El complemento cromosómico desequilibrado con el 15 normal y ausencia de un 13.11,15,17,22 Los gametos no sombreados a la derecha producen descendencia no viable, acontecimientos que explican en la probando(II-7) las pérdidas precoces de los embarazos.

\section{CONCLUSIÓN}

El portador de una translocación Robertsoniana entre los cromosomas 13/15 tiene solo 45 cromosomas, acontecimiento que conduce a la pérdica precoz del embarazo ó al nacimiento de un neonato con mùltiples defectos. En teoría el complemento cromosómico que puede formar un portador en los gametos, son tres viables, uno normal, otro equilibrado, la trisomía 13 y tres gametos desequilibrados que son incompatibles con la vida más allá del inicio del embarazo.

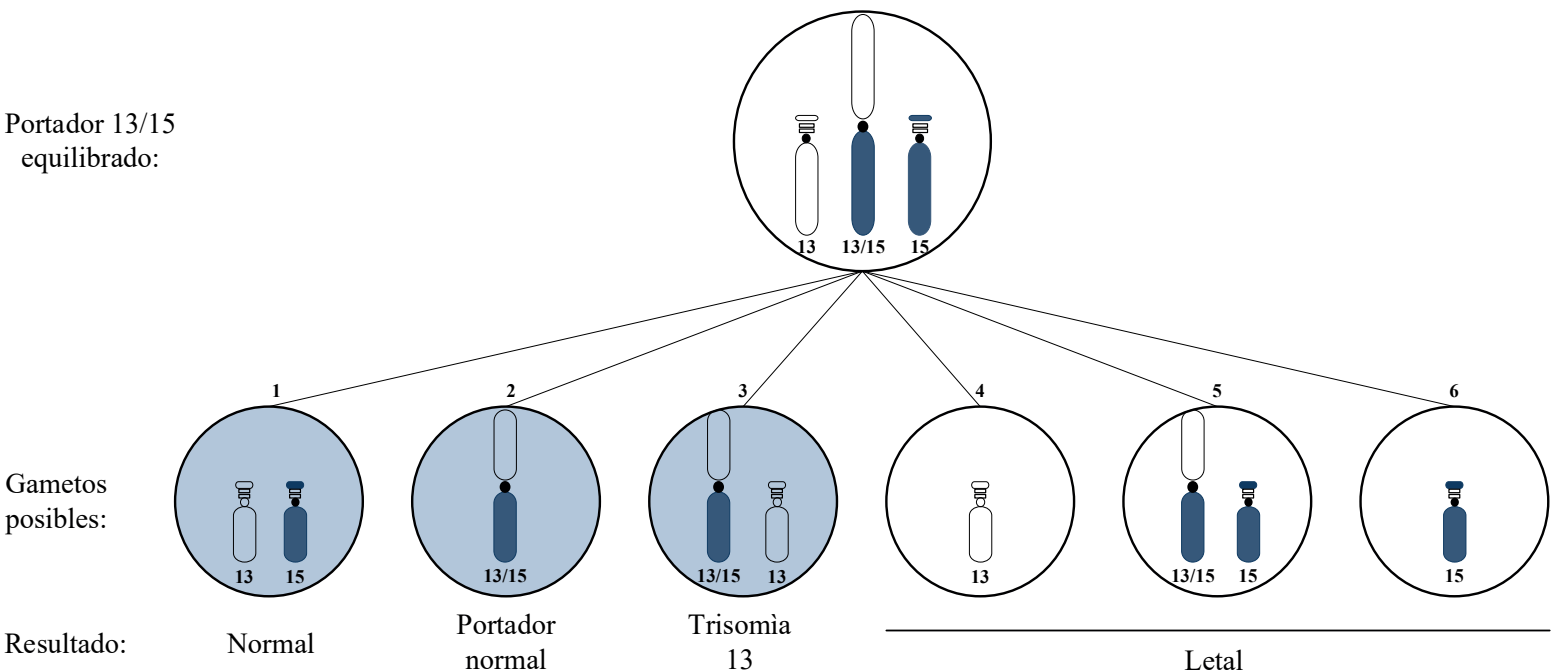

Figura 3. Posibles patrones cromosòmicos de gametos que pueden generarse en la meiosis I 


\section{REFERENCIAS BIBLIOGRÁFICAS}

1. Abdalla EM, Kholeif SF, Elshaffie RM. Homozygosity for a Robertsonian Translocation (13q; 14q) in an Otherwise Healthy 44, XY Man With a History of Repeated Fetal Losses. Lab Medicine. 2013; 44 (3): 254-7.

2. Wang B, Xia Y, Song J, Wang W, Tang Y. Case Report: Potential speciation in humans involving robertsonian translocations. Biomed Res. 2013; 24(1): 171-4.

3. Online Mendelian Inheritance in Man-OMIM. 2016. Disponible en http://www.ncbi.nlm.nih. gov/Omim/. Acceso noviembre 2016.

4. Song J, Li Xi, Sun Lei, Xu S, Liu N, Yao Y. et al. A family with Robertsonian translocation: a potential mechanism of speciation in humans. BioMed Central. 2016; 1(2):1-7

5. Miryounesi M, Diantpour M, Motevaseli E, Chafouri-Fard S. Homozygosity for a Robertsonian Translocation (13q;14q) in a Phenotypically Normal 44, XX female with a history of recurrent abortion and a normal pregnancy outcome. J Reprod Infertil. 2016; 17(3): 184-7.

6. Solari A. Fundamentos y aplicaciones en medicina: Genética humana. 3a ed. Bogotá: Editorial Médica Panamericana; 2008.

7. Slovak M, Theisen Aaron, Shaffer. Human chromosome nomenclature: An overview and definition of terms. En: Gersen S, Keagle M. The principles of clinical cytogenetic. 3a rev ed. New York: Springer Science; 2013; p.34-6.

8. Cruz M, Bosh J. Atlas de Síndromes pediátricos. Barcelona: ESPAXS; 2008.

9. Bacolla A, Wells R. Non-B DNA and chromosomal rearrangements. En: Lupski J, Stankiewicz P. The genomic basis of disease. New Jersey. ANSI. 2006. p.93-4.
10. Lyons Kenneth. SMITH Patrones reconocibles de malformaciones humanas. 6a ed. Barcelona: Elsevier Saunders; 2008.

11. Strachan T, Read A. Genética Humana. 3르 ed. New York: McGraw-Hill Interamericana; 2011.

12. Ecuador. Ministerio de Salud Pùblica. Diagnóstico y tratamiento del aborto espontàneo, incompleto diferido y recurrente: guía de pràctica clínica.Quito: [s.n.]; 2013.

13. Lantigua A. Introducción a la Genética Médica. 2a ed. La Habana: Ciencias Médicas; 2011.

14. Luque J, Herràez A. Biología molecular e ingeniería genética: conceptos, técnicas y aplicaciones en ciencias de la salud. La Habana. Ciencias Médicas. 2001. p. 406-10.

15. Turnpenny P, Ellard S. Emery's Elements of Medical Genetics. 13va ed. Edimburgo: Elsevier Limited; 2007.

16. Paz-Y-Mino C, López-Cortés A. Genética molecular y citogenética humana: fundamentos, aplicaciones e investigaciónes en el Ecuador. Quito. Yachay. 2014. p.245-7.

17. Nussbaum R, Mclnnes R, Willard H. Thompson-Thompson Genética Médica. $7 \mathrm{ma}$ ed. Barcelona; Elsevier Masson; 2009.

18. Yip MY. Uniparental disomy in Robertsonian translocations: strategies for uniparental $\mathrm{di}^{-}$ somy testing. Transl Pediatr. 2014; 3(2): 98-107.

19. Hasanzadeh-Nazar M, Baghbani F, Namazi I, Mirzaee S. Robertsonian translocation between chromosomes ( $n^{\circ}$ 21/14) in relation to the history of spontaneous abortion in a family. J Reprod Med. 2014; 12(8): 581-5. 